\title{
Successfully controlling malaria in South Africa
}

\author{
L Blumberg, ${ }^{1,2}$ MMed (Path Microbiol); J Frean ${ }^{1,3}$ MMed (Path Microbiol); D Moonasar, ${ }^{4}$ DrPH; \\ and the South African Malaria Elimination Committee ${ }^{5}$ \\ ' National Institute for Communicable Diseases, Johannesburg, South Africa \\ ${ }^{2}$ Division of Clinical Microbiology, Faculty of Health Sciences, University of the Witwatersrand, Johannesburg, South Africa \\ Wits Research Institute for Malaria, Faculty of Health Sciences, University of the Witwatersrand, Johannesburg, South Africa \\ ${ }^{4}$ National Department of Health, Pretoria, South Africa \\ ${ }^{5}$ C Asomugha, L Baker, KI Barnes, L Blumberg, B D Brooke, M Coetzee, T de Jager, C Duvenhage, J Frean, M A Groepe, I Kleinschmidt, L L Koekemoer, \\ P Kruger, A Mabuza, R Maharaj, B Margo, N T Mayet, E Misiani, R Mokate, D Moonasar, N Morris, J Raman, E Raswiswi, IS Ukpe
}

Corresponding author: L Blumberg (lucilleb@nicd.ac.za)

Following major successes in malaria control over the past 75 years, South Africa is now embarking on a malaria elimination campaign with the goal of zero local transmission by the year 2018. The key control elements have been intensive vector control, primarily through indoor residual spraying, case management based on parasitological diagnosis using evidence-based drug policies with artemisinin-based combination therapy since 2001, active health promotion in partnership with communities living in the malaria transmission areas, and cross-border collaborations. Political commitment and long-term funding for the malaria control programme have been a critical component of the programme's success. Breaking the cycle of transmission through strengthening of active surveillance using sensitive molecular tests and field treatment of asymptomatic persons, monitoring for antimalarial drug resistance and insecticide resistance, strengthening cross-border initiatives, and ongoing programme advocacy in the face of a significant decrease in disease burden are key priorities for achieving the elimination goal.

S Afr Med J 2014;104(3 Suppl 1):224-227. DOI:10.7196/SAMJ.7600

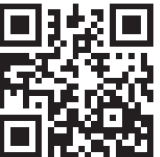

Because of its geographical location at the southernmost extent of malaria distribution, historically South Africa (SA) was spared much of the devastation caused by malaria in the rest of Africa. Nevertheless, seasonal malaria epidemics caused many deaths until the advent of effective vector control methods and antimalarial drugs in the 1930s and 1940s. The country has recently managed to turn the tide on this disease by implementing practical, evidence-based, World Health Organization (WHO)-recommended interventions. Today the programme has moved beyond malaria control and is on a trajectory for malaria elimination (defined as zero local malaria cases in a defined geographical area). ${ }^{[1]}$ In this article we document the successes and challenges that SA has experienced in malaria control and highlight priorities for elimination of the disease.

There were 219 million cases of malaria in 2010 and an estimated 660000 deaths globally, with Africa being the most affected continent, contributing $\sim 90 \%$ of all malaria deaths. ${ }^{[2]}$ Several declarations committing to reducing the burden of malaria have been signed by African heads of state and ministers. The Abuja Declaration was signed on 25 April 2000 by leaders from 44 malaria-endemic countries, in which they committed to an intensive effort to halve malaria mortality for African people by 2010, from the baseline of the year 2000. ${ }^{[3]}$ The Millennium Development Goals (MDGs), adopted in 2001, comprise the key goals of alleviating poverty and disease and improving human development. Various governments and nongovernmental and multilateral agencies use the MDGs (eight in total) as a universal yardstick to monitor individual country progress on health performance. Goal 6 of the MDGs was set to combat HIV/ AIDS, malaria and other diseases and has the target of halting these diseases by 2015 and beginning to reverse the incidence of malaria and other infectious diseases. ${ }^{[4]}$
It is important to gauge the progress that SA has made with regard to these declarations. Between 2000 and 2012, SA reduced the burden of malaria by $~ 90 \%$ ( 64622 v. 6846 cases, respectively) and mortality by $\sim 80 \%$ (459 v. 91 deaths, respectively) (Fig. 1). SA has achieved Abuja and MDG targets and is now well poised to pursue a programme of malaria elimination.

At the African Union Conference of Ministers of Health in 2007, member states launched the Africa Malaria Elimination Campaign, committing to transition eligible countries from malaria control to malaria elimination. ${ }^{[5]}$ Later that year, the Southern African Development Community (SADC) similarly pledged to eliminate malaria from southern Africa. The SADC Malaria Strategic Framework and a subsequent Malaria Elimination Framework urged member states to identify potential areas for elimination and to develop national malaria elimination strategic plans. Encouraged by the promise that a co-ordinated regional approach holds for malaria elimination in southern Africa, a concept of the Malaria Elimination 8 was agreed to by SADC member states, committing six countries - Botswana, Namibia, SA and Swaziland, as well as the island states of Zanzibar and Madagascar - to eliminate malaria by $2015{ }^{[6]}$ It is clear that malaria does not respect national borders, and sustainable elimination in SA will require regional involvement. The Lubombo Spatial Development Initiative (LSDI) has shown what can be achieved by regional co-operation, particularly with regard to Swaziland and KwaZulu-Natal and Mpumalanga provinces. The LSDI started in 1999 as a large-scale project and was initiated in northern KwaZulu-Natal, Mpumalanga, southern Mozambique and Swaziland. It is aimed at accelerating development, particularly with regard to tourism, within an area of $\sim 100000 \mathrm{~km}^{2} .{ }^{[7]}$ Subsequent to the robust implementation of an indoor residual spraying (IRS) programme, coupled with definitive diagnosis and treatment with artemesinin-based combination treatments (ACTs), the incidence 
of malaria has declined in SA (KwaZuluNatal and Mpumalanga provinces) and Swaziland (Lubombo) by 99\% compared with the 2000 baseline. In KwaZulu-Natal and Mpumalanga provinces, malaria to 550 cases) and $72 \%$ (from $\sim 12000$ to 3350 cases), respectively, between the $1999 / 2000$ and 2010/2011 malaria seasons. incidence decreased by 99\% (from 42 400

The prevalence of the disease had decreased by $92 \%$ in southern Mozambique up to 2009 (Fig. 2). ${ }^{[8]}$

Unfortunately, international funding for the LSDI has dried up and it is likely that some of the gains achieved in Mozambique will not be sustained. Notwithstanding this, SA is embarking on a campaign to eliminate the disease within its borders by $2018 .^{[9]}$

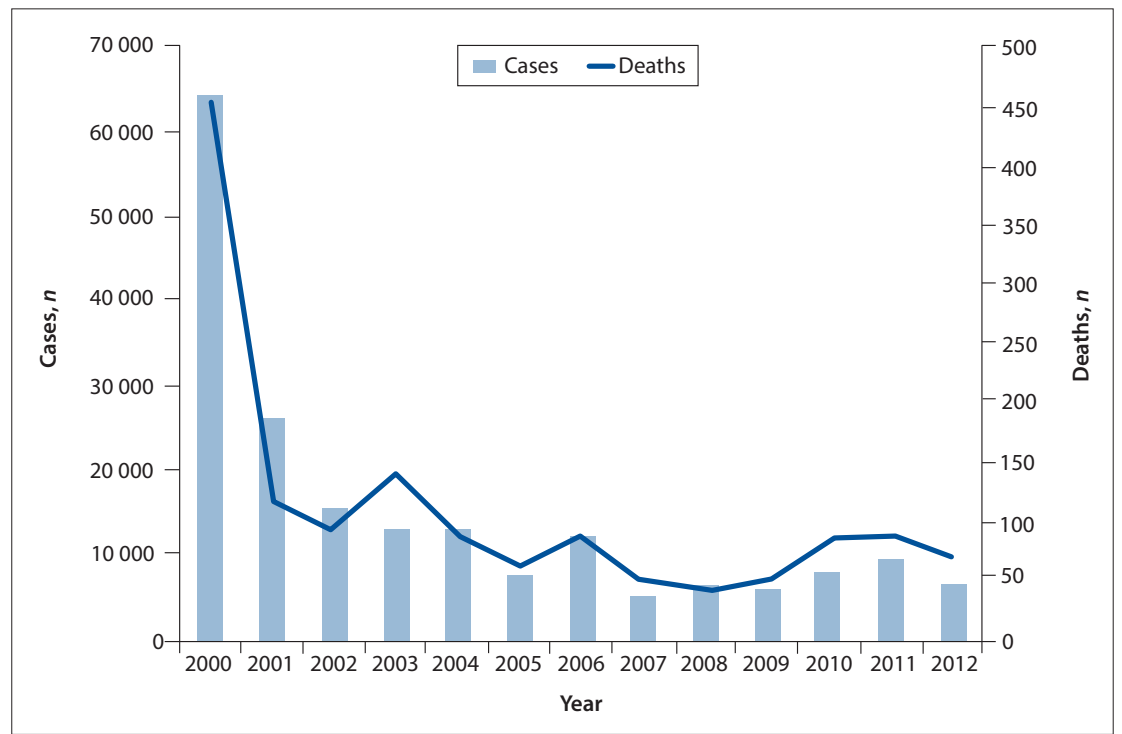

Fig 1. Malaria cases and deaths, South Africa, 2000 - 2012.

\section{From control to elimination: The key elements}

For SA to stay on target for eliminating malaria, the following are essential: ${ }^{[0]}$

- active surveillance in response to confirmed cases and treatment of identified cases, to interrupt local transmission

- utilising sensitive diagnostic tests to detect low-level parasitaemia

- maintaining a high level of malaria awareness in communities and health workers, as the malaria prevalence decreases

- monitoring parasite drug and vector resistance, as these risks increase when malaria case numbers decrease

- supporting malaria control measures in neighbouring countries more effectively

- sustaining continued resourcing in the face of reduced case numbers.

\section{Malaria vector control}

In a malaria vector control programme, accurate identification by modern molecular methods of the mosquitoes involved in transmission of the disease is essential. Mosquitoes are incriminated in malaria transmission by detecting Plasmodium sporozoites or circumsporozoite protein in identified females with immunological and

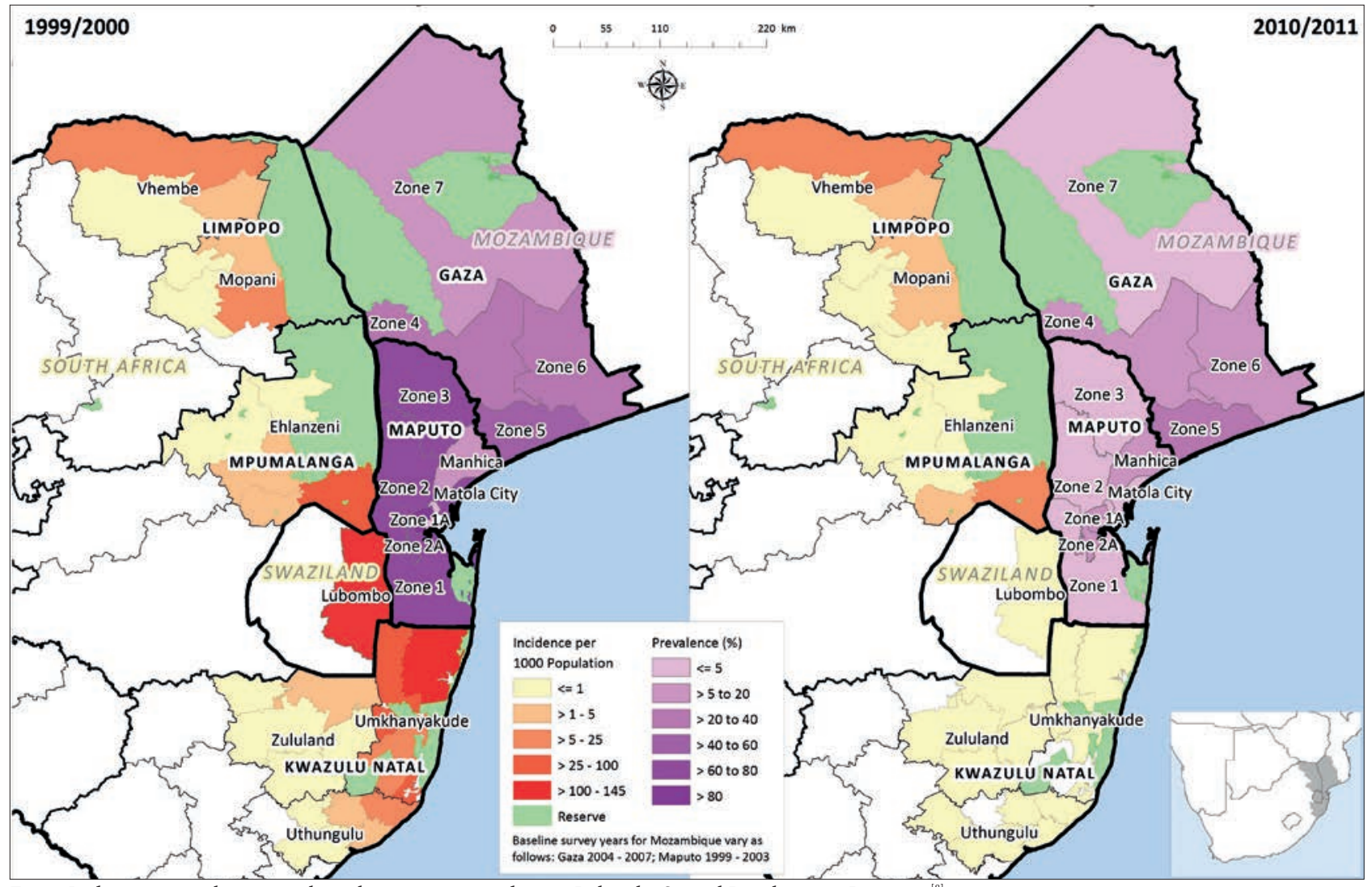

Fig. 2. Reduction in malaria prevalence between 2000 and 2010, Lubombo Spatial Development Initiative. ${ }^{[8]}$ 
molecular assays. In SA, in general, Anopheles funestus is usually associated with outbreaks and epidemics, while A. arabiensis is considered largely responsible for comparatively low-level seasonal transmission in malaria-affected regions. ${ }^{[10]}$ Malaria vector control is insecticide based, relying primarily on IRS. This intervention has generally proved effective where appropriately implemented..$^{[1,12]}$ However, insecticide resistance in target vector populations can undermine the effectiveness of IRS and long-lasting insecticide-treated bed nets. ${ }^{[1-16]}$ Resistance to insecticides in SA malaria vector populations is a relatively recent occurrence. In 1999 - 2000, a malaria epidemic in KwaZulu-Natal Province erupted because pyrethroid resistance caused failure of IRS, and led to the re-introduction of dichloro-diohenyltrichloroethane (DDT) for this purpose, under an exemption from the UN Stockholm Convention on Persistent Organic Pollutants, through which DDT had been banned worldwide except for public health use. Together with a treatment policy shift, this change brought the malaria epidemic under control. Insecticide resistance in target vector populations coupled with environmental and health concerns associated with insecticide use has led to a burgeoning interest in the development of alternative forms of vector control ${ }^{[17]}$ as well as to implementation of integrated vector management strategies. ${ }^{[18,19]}$ Entomological surveillance is a fundamentally important activity in a malaria vector control programme, for several reasons, and operational research and specialised diagnostics arise from activities such as species identification using morphological and molecular methods, vector incrimination using immunological methods, and insecticide susceptibility testing.

\section{Case management: Diagnosis}

Accurate diagnosis of malaria is important for the individual patient as well as for control programmes. Microscopic examination of Giemsa-stained blood films is still the operational gold standard for the diagnosis of clinical malaria, but microscope quality and maintenance, and staining quality and technique, are often suboptimal, especially in remote, poorly resourced settings. ${ }^{[20]}$ The malaria rapid diagnostic test (RDT) is relatively simple to perform, and allows for point-of-care diagnosis and hence immediate malaria treatment. ${ }^{[21,2]}$ The SA National Department of Health became the first African health ministry to implement a definitive malaria diagnosis policy using RDTs at all public sector health facilities in 1996. ${ }^{[23]}$ Although RDTs have proved to be extremely cost-effective in sub-Saharan Africa, ${ }^{[2]}$ their usefulness on the continent is sometimes constrained by sensitivity and specificity issues, as well as user-related problems. Typical parasite loads in geographical areas targeted for malaria elimination are expected to fall well below 100 parasites $/ \mu \mathrm{l}$ in blood, the approximate design detection limit of RDTs, fuelling calls for the development and use of more sensitive nucleic acidbased techniques. ${ }^{[25]}$ Microscopy and RDTs, when performed and interpreted optimally, are suitable for case management, and in the local context attention will need to be paid to quality improvement,

Table 1. Year in which first-line malaria treatment policy was changed in South Africa, by province ${ }^{[35]}$

\begin{tabular}{lllll}
\hline & \multicolumn{3}{c}{ Artesunate- } \\
Province & CQ & SP & SP & AL \\
\hline KwaZulu-Natal & $1940 \mathrm{~s}$ & 1988 & - & 2001 \\
Mpumalanga & $1940 \mathrm{~s}$ & 1997 & 2001 & 2006 \\
Limpopo & $1940 \mathrm{~s}$ & 1998 & - & 2004 \\
CQ = Chloroquine; $\mathrm{SP}=$ sulphadoxine-pyrimethamine; $\mathrm{AL}$ & = artemether-lumefantrine.
\end{tabular}

maintenance and support. As SA embarks on its elimination agenda, mass screening and treating sufficient numbers of potential malaria carriers, whether symptomatic or asymptomatic, to interrupt malaria transmission becomes imperative. ${ }^{[2]}$ Detecting and eradicating every parasite, both asexual parasites and gametocytes, using methods sensitive to low-density infections such as nucleic acidbased technologies, is therefore essential. ${ }^{[2,27]}$ Efficient surveillance of parasite drug resistance by in vitro, in vivo, and/or molecular methods will be essential to inform treatment policy.

\section{Case management: Treatment}

South Africans living in malaria transmission areas are generally nonimmune and all age groups are at risk of developing severe malaria. ${ }^{[28,29]}$ Prompt treatment with effective drugs is the key to reducing malaria morbidity and mortality. ${ }^{[1,30,3]}$ Evidence-based guidelines have dictated the selection of antimalarials in the SA malaria control programme, and have also considered the impact on transmission and antimalarial drug resistance issues.

Before the advent of chloroquine (CQ) in the late 1940s, quinine was used for both treatment and prophylaxis. During the 1970s, the focus was on malaria case detection and CQ treatment, mainly through active case finding during house-to-house surveys and mass blood examination. In the mid-1990s, the District Health System, based on primary healthcare, was adopted as the healthcare strategy for SA. ${ }^{[32]}$ Passive detection and prompt, effective treatment of malaria at primary healthcare facilities, based on parasitological diagnosis with RDTs, became the policy for case management. ${ }^{[3,34]}$

The emergence of CQ resistance in KwaZulu-Natal necessitated a change to sulphadoxine-pyrimethamine (SP) in 1988, and then to artemether-lumefantrine (AL) in 2001, following treatment failures and identification of molecular resistance markers for SP. Treatment policy changes in Limpopo and Mpumalanga provinces were based on rigorous drug resistance monitoring and early identification of predictive molecular resistance markers for SP resistance (Table 1). ${ }^{[35]}$

$\mathrm{AL}$ is the current first-line antimalarial for the treatment of uncomplicated falciparum malaria. Second-line antimalarials for uncomplicated malaria remain oral quinine, plus doxycycline (for adults) or clindamycin (for pregnant women and children $<8$ years). ${ }^{[2]}$ Parenteral quinine is the mainstay of treatment of severe malaria. ${ }^{[3,37]}$ Intravenous artesunate is currently recommended by the WHO for severe malaria in adults and children, with an increased survival advantage and fewer adverse reactions than quinine. ${ }^{[30,38]}$ Intravenous artesunate is not yet registered for use in SA, but there is limited availability through a special access programme. ${ }^{[29]}$

The number of malaria-related deaths is a key epidemiological indicator to evaluate performance in delivering effective malaria case management. ${ }^{[30]}$ Late presentation, lack of awareness of malaria in communities, and a low index of suspicion on the part of healthcare workers, particularly in non-endemic provinces in the country, ${ }^{[39-42]}$ are major factors contributing to malaria-related deaths. Even in the endemic provinces, case fatality rates (number of deaths per 100 cases of malaria) are above the national target of $<0.5 \%$. Continuing education of healthcare workers on malaria diagnosis and treatment is of critical importance. ${ }^{[43]}$

The emergence of drug resistance in areas of low transmission intensity is the biggest threat to treatment. With artemisinin resistance already confirmed in the Greater Mekong Region of Southeast Asia, and no molecular marker for detecting artemisinin-resistant parasites, there is a real possibility of this resistance spreading to, or emerging in, SA. Conducting in vivo drug efficacy trials in regions approaching malaria elimination is virtually impossible owing to low case numbers. Until validated molecular markers are available 
for artemisinin resistance, our best options are routine monitoring for molecular markers of resistance to lumefantrine (or future ACT partner drugs), and whenever possible to test for $P$. falciparum parasites 3 days after treatment, and to follow up patients 4 - 6 weeks after treatment to establish whether they remain malaria free. ${ }^{[4]}$

Interrupting the cycle of malaria transmission in the community is a key strategy in the continuum from control to elimination. Active case surveillance using sensitive molecular techniques, the use of gametocytocidal drugs such as primaquine, ${ }^{[45]}$ and communitybased treatment of asymptomatic malaria all need to be considered. Before the elimination programme decides to go ahead with routine primaquine use, it needs to update local knowledge about glucose-6phosphate dehydrogenase deficiency in SA, for ethical and scientific reasons. ${ }^{[46]}$

\section{Conclusions}

SA has achieved remarkable success in controlling malaria over the past century, and particularly in the past decade. Epidemiologically the incidence of malaria in SA places the country in the preelimination category, namely $<5$ malaria cases per 1000 population at risk, and in some municipalities at the elimination stage of $<1$ case per 1000 population at risk. ${ }^{[1]}$ However the success of SA's malaria elimination programme will depend on early detection of malaria cases, with rapid appropriate treatment and vector control response to foci of transmission, maintaining a high level of vector control coverage, especially in the high-transmission areas, ensuring that drugs, insecticides and diagnostic tools are of the highest quality, ensuring that cross-border malaria initiatives are ongoing, monitoring insecticide and drug resistance, and sustaining partnerships in advocating for continued resourcing for malaria control and elimination.

Acknowledgement. We thank D N Durrheim, F Hansford, K W Hlongwana, R Hunt, H Jooste, B Poonsamy, I Seocharan, B Shandukani, and J Urbach for many years of invaluable technical contributions to the malaria control programme in SA.

\footnotetext{
References

1. World Health Organization. Malaria Elimination: A Field Manual for Low and Moderate Endemic Countries. Geneva: WHO, 2007. http://www.who.int/iris/handle/10665/43796 (accessed 20 January 2014). 2. World Health Organization. World Malaria Report: 2012. Geneva: WHO, 2012. http://www.whoint/ iris/handle/10665/78945 (accessed 20 January 2014).

3. World Health Organization. The Abuja Declaration and the Plan of Action. Geneva: WHO, 2003 http://www.rollbackmalaria.org/docs/abuja_declaration.pdf (accessed 20 January 2014).

4. World Health Organization. Millenium Development Goals. Geneva: WHO, 2000. http://www.who.int/ 4. World Health Organization. Millenium Development Goals. Geneva: WHO, 2000 .
topics/millennium_development_goals/about/en/ (accessed 20 January 2014).

5. African Union Secretariat. Fight Malaria: Africa Goes From Control to Elimination by 2010. 3rd Sesssion African Union Secretariat. Fight Malaria: Africa Goes From Control to Elimination by 2010. 3rd Sesssion
of the AU Conference of Ministers of Health, Johannesburg, 2007. http://www.aidswatchafrica.org/sites default/files/publication-documents/AU_Malaria_Camapign.pdf (accessed 20 January 2014).

6. SADC Secretariat. Malaria Elimination. http://tis.sadc.int/english/sarn/elimination-eight-e8/ (accessed 20 January 2014).

7. Sharp BL, Kleinschmidt I, Streat E, et al. Seven years of regional malaria control collaboration Mozambique, South Africa, and Swaziland. Am J Trop Med Hyg 2007;76(1):42-47.

8. South African Medical Research Council. Annual Report 2010/2011. http://www.mrc.ac.za/ annualreport/annualreport1011.pdf (accessed 20 January 2014).

9. National Department of Health. Malaria Elimination Strategy for South Africa 2012-18. Pretoria: $\mathrm{NDoH}, 2012$

10. Coetzee M, Hunt RH. Malaria at its southern-most fringe in Africa. Research and Reviews in

Parasitology 1998;58(3/4):175-179.
}

11. World Health Organization. Global Insecticide Use for Vector-borne Disease Control. Geneva: WHO, 2011. http://www.who.int/iris/handle/10665/44670 (accessed 11 August 2013).

12. Pluess B, Tanser FC, Lengeler C, Sharp BL. Indoor residual spraying for preventing malaria. Cochrane Database Syst Rev 2010;(4):CD006657. [http://dx.doi.org/10.1002/14651858.CD006657.pub2]

13. Govere JM, Durrheim DM, Kunene S. Malaria trends in South Africa and Swaziland and the introduction of synthetic pyrethroids to replace DDT for malaria control. S Afr J Sci 2002;98(1-2):19-21.

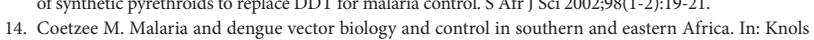
Coetzee M. Malaria and dengue vector biology and control in southern and eastern Africa. In: Knols
BGJ, Louis C, eds. Bridging Laboratory and Field Research for Genetic Control of Disease Vectors. Wageningen: UR Frontis Series No. 11, 2005:101-109. [http://dx.doi.org/10.1007/1-4020-3799-6_9]

Wageningen: UR Frontis Series No. 11, 2005:101-109. [http://dx.doi.org/10.1007/1-4020-3799-6_9]
15. N'Guessan R, Corbel V, Akogbéto M, Rowland M. Reduced efficacy of insecticide-treated nets and indoor residual spraying for malaria control in pyrethroid resistance area, Benin. Emerg Infect Dis 2007;13(2):199-206. [http://dx.doi.org/10.3201/eid1302.060631]

16. Ranson H, N'guessan R, Lines J, Moiroux N, Nkuni Z, Corbel V. Pyrethroid resistance in African anopheline mosquitoes: What are the implications for malaria control? Trends Parasitol 2011;27(2):9198. [http://dx.doi.org/10.1016/.jpt.2010.08.004]

17. Takken W, Knols BG. Malaria vector control: Current and future strategies. Trends Parasitol 2009;25(3):101-104. [http://dx.doi.org/10.1016/j.pt.2008.12.002]

18. World Health Organization. Global Strategic Framework for Integrated Vector Management. Document WHO/CDS/CPE/PVC/2004.10. Geneva: WHO, 2004. http://www.who.int/iris/handle/10665/68624 (accessed 11 August 2013).

19. Chanda E, Masaninga F, Coleman M, et al. Integrated vector management: The Zambian experience. Malar J 2008; 7:164. [http://dx.doi.org/10.1186/1475-2875-7-164]

20. Zikusooka CM, McIntyre D, Barnes KI. Should countries implementing an artemisinin-based combination malaria treatment policy also introduce rapid diagnostic tests? Malar J 2008;7:176. [http://dx.doi.org/10.1186/1475-2875-7-176]

21. Gitonga CW, Kihara JH, Njenga SM, et al. Use of rapid diagnostic tests in malaria school surveys in Kenya: Does their under-performance matter for planning malaria control? Am J Trop Med Hyg 2012;87(6):1004-1011. [http://dx.doi.org/10.4269/ajtmh.2012.12-0215]

22. Moonasar D, Goga AE, Frean J, Kruger P, Chandramohan D. An exploratory study of factors that affect the performance and usage of rapid diagnostic tests for malaria in the Limpopo Province, South Africa. Malar J 2007;6:74. [http://dx.doi.org/10.1186/1475-2875-6-74]

23. Barnes KI, Durrheim DN, Little F, et al. Effect of artemether-lumefantrine policy and improved vector control on malaria burden in KwaZulu-Natal, South Africa. PLoS Med 2005;2(11):e330. [http://dx.doi. org/10.1371/journal.pmed.0020330]

24. Shillcutt S, Morel C, Goodman C, et al. Cost-effectiveness of malaria diagnostic methods in subSaharan Africa in an era of combination therapy. Bull World Health Organ 2007;86(2):101-110. [http:// dx.doi.org/10.2471/BLT.07.042259]

25. malERA Consultative Group on Diagnosis and Diagnostics. A research agenda for malaria eradication: Diagnoses and diagnostics. PLoS Med 2011;8(1):e1000396. [http://dx.doi.org/10.1371/journal. pmed.1000396]

26. Rheeder JC, Targett GA, Shanks D, Greenwood B. Killing the Parasite. San Francisco: Global Health Group, 2009:13.

27. McMorrow ML, Aidoo M, Kachur SP. Malaria rapid diagnostic tests in elimination settings - can they find the last parasite? Clin Microbiol Infect 2011;17(11):1624-1631. [http://dx.doi.org/10.1111/j.14690691.2011.03639.x]

28. Blumberg L, Frean J. Malaria control in South Africa - challenges and successes. S Afr Med J 2007;97(11):1193-1197.

29. National Department of Health. Guidelines for the Treatment of Malaria in South Africa. Pretoria: $\mathrm{NDoH}, 2010$

30. World Health Organization. Malaria Case Management Operations Manual. Geneva: WHO, 2009. http://www.who.int/iris/handle/10665/44124 (accessed 12 August 2013).

31. World Health Organization. Guidelines for the Treatment of Malaria. 2nd ed. Geneva: WHO, 2010. horlt://www.who.int/iris/handle/10665/44227 (accessed 12 August 2013).

32. Health Systems Trust. Bringing Health Closer to People: Local Government and the District Health System. Durban: Health Systems Trust, 2001. http://www.hst.org.za/all-publications (accessed 30 July 2013).

33. National Department of Health. White Paper for the Transformation of the Health System in South Africa. Pretoria: NDoH, 1997. http://www.doh.gov.za/index.php (accessed 30 July 2013)

34. Durrheim DN, la Grange JJ, Govere J, et al. Accuracy of a rapid immunochromatographic card test for Plasmodium falciparum in a malaria control programme in South Africa. Trans R Soc Trop Med Hyg 1998;92(1):32-33. [http://dx.doi.org/10.1016/S0035-9203(98)90944-0]

35. Moonasar D, Nuthulaganti T, Kruger PS, et al. Malaria control in South Africa 2000-2010: Beyond MDG6. Malar J 2012;11:294. [http://dx.doi.org/10.1186/1475-2875-11-294]

36. Achan J, Talisuna AO, Erhat A, et al. Quinine, an old anti-malarial drug in a modern world: Role in the treatment of malaria. Malar J 2011;10:144. [http://dx.doi.org/10.1186/1475-2875-10-144]

37. World Health Organization. Global Antimalarial Drug Policies Database - WHO African Region December 2012 Update. Geneva: WHO, 2012.

38. World Health Organization. Guidelines for the Treatment of Malaria. 2nd ed., rev. 1. Geneva: WHO, 2011

39. Spencer-Jones JH. Malaria update. S Afr Med J 1996;86(6):622-624.

40. Soni PN, Gouws E. Severe and complicated malaria in KwaZulu-Natal. S Afr Med J 1996;86(6):653-656.

40. Soni PN, Gouws E. Severe and complicated malaria in KwaZulu-Natal. S Afr Med J 1996;86(6):653-656.
1. Durrheim DN, Frieremans S, Kruger P, et al. Confidential inquiry into malaria deaths. Bull World Health Organ 1999;77(3):263-266

42. Mehta U, Durrheim DN, Blumberg L, et al. Malaria deaths as sentinel events to monitor healthcare delivery and antimalarial drug safety. Trop Med Int Health 2007;12(5):617-628. [http://dx.doi. org $/ 10.1111 / j .1365-3156.2007 .01823 . x]$

43. Ukpe IS. Continuing medical education in unstable malaria areas. Bull World Health Organ 1999;77(11):948.

4. Stepniewska K, Ashley E, Lee SJ, et al. In vivo parasitological measures of artemisinin susceptibility. J Infect Dis 2010;201(4):570-579. [http://dx.doi.org/10.1086/650301]

45. World Health Organization. The Safety and Effectiveness of Single Dose Primaquine as a $P$. falciparum Gametocytocide. WHO Evidence Review Group. Geneva: WHO, 2012

46. Beighton P, Botha MC. Inherited disorders in the black population of southern Africa. Part I. Historical and demographic background; genetic haematological conditions. S Afr Med J 1986;69(4):247-249. 